\title{
ON A FUNDAMENTAL FORMULA IN THE THEORY OF CLASS-NUMBER RELATIONS*
}

BY E. T. BELL

1. The Fundamental Formula. In a series of memoirs Sur les relations entre les nombres des classes des formes quadratiques et positives written about seven years ago but only now in course of publication, $\dagger$ Professor J. Ouspensky, perfecting Liouville's unpublished method for class-number relations (Liouville stated only his results), obtains by simple, uniform processes applied to a single formula "not only all the relations hitherto known, with the possible exception of the very general results of Hurwitz, but also many others which appear to be new." The formula in question is (loc. cit., p. 604),

$$
\begin{gathered}
\sum[f(d+\delta, \nu, d-\delta)-2 f(\delta-2 \nu, d+\nu, 2 d-\delta+2 \nu)] \\
=\epsilon(m) \sum_{j=1}^{2 m^{1 / 2}-1}\left[\begin{array}{c}
f\left(2 m^{1 / 2}, m^{1 / 2}-j, 2 m^{1 / 2}-2 j\right) \\
\left.-2 f\left(2 m^{1 / 2}-j, m^{1 / 2}, 2 m^{1 / 2}-j\right)\right]
\end{array}\right.
\end{gathered}
$$

where $\sum$ on the left refers to all integers $\nu \gtreqless 0, d>0, \delta>0$ such that, for $m$ an arbitrary constant integer $>0$,

$$
m=\nu^{2}+d \delta
$$

$\epsilon(t)=1$ or 0 according as $t$ is or is not the square of an integer $>0$, and $f(x, y, z)$ is a single-valued function whenever its arguments are simultaneously integers $\gtreqless 0$, subject to the parity conditions

$$
\left\{\begin{array}{l}
f(-x, y, z)=-f(x, y, z), \quad f(0, y, z)=0 \\
f(x,-y,-z)=f(x, y, z)
\end{array}\right.
$$

beyond which $f$ is entirely arbitrary.

* Presented to the Society, San Francisco Section, June 12, 1926.

† Bulletin DE L'ACademie des Sciences DE RUSSIE, 1925, pp. 599-620, 763-784; ibid., 1926, pp. 25-38, 175-196, 327-348. 
It is truly remarkable that an identity so simple as (1) should unite a great body of heterogeneous theorems, many of which were first found by abstruse analysis, and reveal their common origin in a matter of elementary arithmetic. In a further Note sur le nombre des représentations des nombres par une somme d'un nombre de carrés, ${ }^{*}$ the author announces that the same "formule vraiment fondamentale" yields also the general expression of Boulyguine $\dagger$ for the number of representations of a given integer by any even number of squares, and he gives the extremely simple developments for $2,4,6,8,10,12$ squares. Further, as remarked by the author, it is evident to anyone conversant with this field that the applications of (1) to class-number formulas and to representations as sums of squares are but a small part of the specific arithmetic facts implicit in the general identity.

Professor Ouspensky's proof of (1) is purely arithmetic and leaves nothing to be desired in the way of elementary simplicity. Believing however that even an elementary proof by strictly arithmetic methods is of a far higher order of difficulty, because such a proof is in general a thing in itself with but slight indications of possible generalizations, than is an algebraic proof employing more advanced analysis, I shall offer in $\S 2$ a proof of (1) of the latter kind. $\ddagger$ This proof, equally as elementary from the algebraic point of view as is the author's from the arithmetic, refers the theorem to membership in an infinite class of allied results, all of

* Ibid., 1925, pp. 647-662. 1914.

† Bulletin de l'Académie des Sciences de St. Pétersbourg, April,

$\ddagger$ It is not here a question of rigor, but of practicability, in agreement with the following remarks by H. J. S. Smith (Collected PAPERs, vol. 1, p. lxxiv) on the formal perfection of Gauss' synthetic proofs: "Every assertion that is made is fully proved; . . . but when we have finished the perusal, we soon begin to feel that our work is but begun . . $\cdot$. No vestige appears of the process by which the result itself was obtained, perhaps not even a trace of the considerations which suggested the successive steps of the demonstration." 
which are immediate consequences of Jacobi's formula for the multiplication of four elliptic theta functions or, if preferred, of the equation of three terms in elliptic functions, combined with the arithmetic expansions of the doubly periodic functions of the second kind (Hermite's terminology). It is a striking fact that Ouspensky's farreaching theorem is one of the very simplest of all those concerning arbitrary odd or even functions in more than two variables which are implied by Jacobi's theta formula, and which may be elicited from it by the most elementary algebraic considerations.

In a paper not yet published concerning what may be called the inverse method of paraphrases* I have shown that any theorem whatever relating to arbitrary odd or even functions in any number of variables of the same general character as (1), in which the variables are linear functions of the indeterminates representing a set of integers in a system of quadratic forms, is implied by Jacobi's theta formula and the expansions of the doubly periodic functions of the second kind. The doubly periodic functions of the $r$ th kind, $r>1$, also lead to similar results; $r$ determines the type of arithmetic functions occurring as coefficients of the arbitrary functions; for any $r$ the theorems are of essentially one kind, viz., they relate to functions of integers arbitrary except as to parity. I have further shown, in the paper mentioned, how any given result of this kind can be traced immediately by a straightforward, uniform method to its simplest equivalent identity in theta functions. The proof in $\S 2$ was constructed in this way; it will be sufficient here to give the proof directly. Having obtained such an algebraic proof of any theorem we can then extend the theorem indefinitely by multiplying its generating theta identity by functions of theta functions or of circular functions, and reducing, or by numerous other devices of

* For the direct method, used below in $\$ 2$, see TRANSACtions of THIS SOcIETY, vol. 22 (1921), p. 1. 
e lementary algebra that at once suggest themselves. This is the principal advantage of the inverse method; from the proof by its means of a known theorem the given result can be extended at will. It thus appears that any such result as (1) is included as a special case in each of an infi nity of similar theorems relating to functions of $4,5,6, \ldots$ variables. Conversely, starting from any theta identity we can paraphrase it directly, and by specializing the arbitrary functions in the result, deduce an indefinite number of specific arithmetic theorems. Thus the two methods of paraphrase, the inverse and the direct, are complementary.

2. Algebraic Proof of (1). In the identity

take

$$
\left|\begin{array}{lll}
a_{3} & b_{3} & c_{3} \\
a_{3} & b_{3} & c_{3} \\
a_{2} & b_{2} & c_{2}
\end{array}\right|=0
$$

$$
a_{i}=\vartheta_{j}(2 x+y+2 z), \quad b_{j}=\vartheta_{j}(2 x-y-2 z), \quad c_{j}=\vartheta_{j}(y),
$$

and use

$\vartheta_{1}(u) \vartheta_{1}(v)=\vartheta_{3}\left(u+v, q^{2}\right) \vartheta_{2}\left(u-v, q^{2}\right)-\vartheta_{2}\left(u+v, q^{2}\right) \vartheta_{3}\left(u-v, q^{2}\right)$

to reduce the result. Then, dividing throughout by

$$
\vartheta_{1}\left(x+z, q^{1 / 2}\right) \vartheta_{1}\left(x-z, q^{1 / 2}\right) \vartheta_{1}\left(y+2 z, q^{1 / 2}\right),
$$

and introducing the doubly periodic function of the second kind indicated by the resulting identity, namely

we find

$$
\phi_{111}(u, v) \frac{\vartheta_{1}^{\prime} \vartheta_{1}(u+v)}{\vartheta_{1}(u) \vartheta_{1}(v)}
$$

$$
\begin{gathered}
\phi_{111}\left(x+z, x-z, q^{1 / 2}\right) \vartheta_{3}(y) \\
=\phi_{111}\left(x-z, y+2 z, q^{1 / 2}\right) \vartheta_{3}(2 z-y-2 z) \\
+\phi_{111}\left(x+z,-y-2 z, q^{1 / 2}\right) \vartheta_{3}(2 x+y+2 z),
\end{gathered}
$$

where it is necessary (as in the theta functions) to indicate that the parameter in $\phi_{111}$ is $q^{1 / 2}$ not $q$ as it is in $\phi_{111}(u, v)$ 
and $\vartheta_{3}(y)$. This is the simplest identity which is formally equivalent to (1), in the sense that each of (1), (5) implies the other. As stated in $\S 1,(4)$ and the proper theta substitution for $a_{j}, b_{j}, c_{j}$ to yield this formal equivalent of (1) are indicated immediately by the inverse method of paraphrase by simple inspection of (1). In a complete exposition of the theory it would be unnecessary to proceed beyond (5) in order to have the proof of (1). Here, however, we must apply the direct method and verify that (5) actually does imply (1). It remains then to substitute in (5) the series for $\vartheta_{3}$,

$$
\vartheta_{3}(u)=\sum q^{\nu^{2}} \cos 2 \nu x,
$$

where $\sum$ refers to all integers $\nu \frac{\geqq}{\Sigma} 0$,

$$
\phi_{111}\left(u, v, q^{1 / 2}\right)=\operatorname{ctn} u+\operatorname{ctn} v+4 \sum q^{n}\left[\sum \sin 2(d u+\delta v)\right]
$$

where the outer $\sum$ refers to all integers $n>0$ and the inner to all pairs $(d, \delta)$ of conjugate divisors $d, \delta>0$ of $n$ such that $d \delta=n$, in order to reach (1) by paraphrasing the resulting trigonometric identity obtained on equating coefficients of $q$.

With $\sum, m, d, \delta$ as in (1), (2), we find at once

$$
\text { (6) }\left\{\begin{array}{l}
2 \sum[\sin 2\{(d+\delta) x+(d-\delta) z\} \cos 2 \nu y \\
-\sin 2\{d x+\delta y-(d-2 \delta) z\} \cos 2 \nu(2 x-y-2 z) \\
-\sin 2\{d x-\delta y+(d-2 \delta) z\} \cos 2 \nu(2 x+y+2 z)] \\
\quad+\operatorname{ctn}(x+z)\left\{\cos 2 m^{1 / 2}(2 x+y+2 z)-\cos 2 m^{1 / 2} y\right\} \\
\quad+\operatorname{ctn}(y+2 z)\left\{\cos 2 m^{1 / 2}(2 x-y-2 z)-\cos 2 m^{1 / 2} y\right\} \\
\left.\left.\quad-\cos 2 m^{1 / 2}(2 x+y+2 z)\right\}\right] .
\end{array}\right.
$$

By directly paraphrasing reduced forms of (6), we can derive immediately seven theorems similar to (1), one of which is (1), for functions of the respective parities* $p\left(1^{2} \mid 1\right)$ p. 2.

* For the $\mathrm{p}(\mid)$ notation, see Transactions of this Society, loc. cit., 
(three theorems), $p\left(0 \mid 1^{3}\right)$ (one theorem), $p(2 \mid 1)$ (three theorems), all of which are contained as special cases in the result for parity $p\left(0 \mid 1^{3}\right)$, which thus appears as the simplest generalization of (1). Our object here being merely the proof of (1), we note that in order to reach (1) all trigonometric products in (6) must be reduced to the type $\sin a x \cos (b y+c z)$, since this is the circular function of $x, y, z$ having the same parity with respect to $x, y, z$ as $f(x, y, z)$ in (1). As all details of the necessary reduction are common routine in the method of paraphrase, we need give only an outline of the successive steps.

On the left of (6) trigonometric products are separated into sums and recombined. Thus the first line becomes

$$
\begin{aligned}
& 2 \sum \sin 2\{(d+\delta) x+(d-\delta) z\} \cos 2 \nu y \\
&= \sum[\sin 2\{(d+\delta) x+\nu y+(d-\delta) z\} \\
&\quad+\sin 2\{(d+\delta) x-\nu y-(d-\delta) z\}],
\end{aligned}
$$

since $d, \delta$ may be interchanged under $\sum$, and this

$$
=2 \sum \sin 2(d \dot{+} \delta) x \cos 2\{\nu y+(d-\delta) z\},
$$

which is in the required form. After similar separation of the second and third lines the sines whose arguments are the respective sums of the given arguments are recombined, and so for the differences. The result is the sum of

$$
\begin{aligned}
& -2 \sum \sin 2(d+2 \nu) x \cos 2\{(\delta-\nu) y+(2 \delta-d-2 \nu) z\}, \\
& -2 \sum \sin 2(d-2 \nu) x \cos 2\{(\delta+\nu) y+(2 \delta-d+2 \nu) z\},
\end{aligned}
$$

and hence it is twice either, since $\nu$ may be replaced by $-\nu$ under $\sum$. To reduce the right of (6) we must first eliminate the cotangents, as always in paraphrasing. For this we combine the differences of cosines into products of sines, obtaining three products of the type ctn $u \sin 2 a u \sin 2 b v$ where $a, b$ are integers $>0$, after changing the limits of summation for $\nu$ ( $-\infty$ to $\infty)$ to 0 to $\infty$, by combining terms 
equidistant from $\nu=0$ in the usual way. To these products we apply

$$
\operatorname{ctn} u \sin 2 a u=\sum_{j=0}^{2 a-1} \cos 2(a-j) u,
$$

separate products $\cos 2(a-j) u \sin b v$ into sums, and proceed as above in reducing the left. Finally, replacing $x, y, z$ by their halves, and noting that the lower limit $j=0$ may be changed to $j=1$ since $j=0$ causes the summand to vanish identically, we find

$$
\begin{aligned}
& \sum[\sin (d+\delta) x \cos \{\nu y+(d-\delta) z\} \\
& \quad-2 \sin (d+2 \nu) x \cos \{(\delta-\nu) y+(2 \delta-d-2 \nu) z\}] \\
& =\epsilon(m) \sum_{j=1}^{2 m^{1 / 2}-1}\left[\sin 2 m^{1 / 2} x \cos \left\{\left(m^{1 / 2}-j\right) y+\left(2 m^{1 / 2}-2 j\right) z\right\}\right. \\
& \left.\quad-2 \sin \left(2 m^{1 / 2}-j\right) x \cos \left\{m^{1 / 2} y+\left(2 m^{1 / 2}-j\right) z\right\}\right] .
\end{aligned}
$$

Noticing that $\nu$ may be replaced by $-\nu$ and that $d, \delta$ may be interchanged in the second term on the left we see that the last identity paraphrases directly into (1).

By bringing to bear on (5) all of the powerful machinery of the theta functions, including the theory of transformation, we can obtain from it an inexhaustible supply of theorems similar to (1), some of which, it is likely, it would be difficult to find by strictly arithmetic methods although all, no doubt, could be proved by such means with the exercise of sufficient ingenuity.

California Institute of Technology 